\title{
Beware of medicalising the fear of uncertainty and death
}

\section{Louisa Polak general practitioner and PhD student}

North Hill Medical Group, Colchester CO1 1DZ, UK

I strongly second Heath's call for clinicians to be more tolerant of uncertainty, and agree with her that this requires courage, particularly given the prevalent culture of blame. ${ }^{1}$

Nobody could disagree with the recommendation that people should be screened for their potential to benefit from risk reducing interventions "only when medical care is appropriate and will produce more benefit than harm." Most clinicians are well aware of the harms of swapping Sontag's "good passport" for a provisional "at risk" one. ${ }^{2-4}$ The snag with Heath's recommendation is that defining and evaluating "benefit" and "harm," and hence deciding when "medical care is appropriate," requires more than just courage and certainly more than just statistical facts: it requires a complex weighing up of preferences. Like her, I would prefer to avoid preventive drugs. I am aware that this inevitably colours the way I talk with patients about screening but try to keep this to a minimum, because the conversation should centre on the patient's preferences, not mine. Some well informed people choose screening; I am concerned that Heath's strongly expressed preference might well translate in practice into a new version of "doctor knows best."
We need to acknowledge that helping individual patients to make decisions that are right for them, in the way that they want to make them, is a complex and difficult task. Trying to help people not to fear death is a major additional challenge. As Heath says, doctors may well not be the best people to meet this challenge. In attempting to meet it, we should beware of increasing the medicalisation that Heath criticises, by extending the remit of "healthcare" to encompass yet more aspects of human life.

Competing interests: None declared.

Heath I. Role of fear in overdiagnosis and overtreatment—an essay by lona Heath. $B M J$ 2014;349:g6123. (24 October.)

Aronowitz R. The converged experience of risk and disease. Milbank Q2009:87:335-542. Frank AW. The wounded storyteller. University of Chicago Press, 1995.

Scott S, Prior L, Wood F, Gray J. Repositioning the patient: the implications of being "at risk." Soc Sci Med 2005;60:1869-79.

Cite this as: BMJ 2014;349:97077

(๑) BMJ Publishing Group Ltd 2014 